\title{
结构感知深度学习的三维形状分类方法
}

\author{
韩丽，朴京钥，兰鹏燕，王晓旻，千冰，佟宇宁，徐圣斯 \\ (辽宁师范大学计算机与信息技术学院 大连 116081) \\ (h1_dlls@dl.cn)
}

\begin{abstract}
摘 要: 为了解决复杂、海量三维模型的形状识别问题, 提出了一种结构感知深度学习的三维形状分类方法. 通过联 合学习三维模型的几何结构和空间结构, 生成具有结构感知的特征向量表示, 该特征向量具有更强的识别力与稳定 性, 在三维形状分类中取得显著的效果. 首先, 提取优化的多尺度热核特征, 并通过 CNN 学习模型, 有效地获取三 维形状的几何结构特征; 其次, 建立多视图卷积学习网络提取三维形状的空间结构特征; 最后, 通过联合优化学习 生成具有结构感知的深度特征表示. 文中采用了 C++, Matlab, TensorFlow 框架实现, 并在公开的三维数据库中进行 了大量实验, 实验结果表明, 文中方法获取的深层结构特征对于复杂拓扑结构、大尺度几何形变的三维形状具有稳定 性；与相关方法对比，在三维形状分类中具有更高的分类精度.
\end{abstract}

关键词: 形状分类; 结构感知; 卷积神经网络; 深度学习

中图法分类号: TP391.41 DOI: 10.3724/SP.J.1089.2021.18280

\section{D Shape Classification Method Based on Shape-Aware Deep Learning}

Han Li, Piao Jingyu, Lan Pengyan, Wang Xiaomin, Yu Bing, Tong Yuning, and Xu Shengsi (School of Computer Science and Information Technology, Liaoning Normal University, Dalian 116081)

\begin{abstract}
In order to solve the problem of complex and massive 3D shapes recognition, we propose a structure-aware deep learning model for 3D shape classification. By learning the geometric structure and spatial structure jointly, we generate a structure-aware deep feature vector, which has stronger discriminative ability and stability on 3D shape classification. Firstly, optimal multi-scale HKS features are extracted to construct discerning geometric shape descriptor based on a CNN learning model. Secondly, a multi-view based CNN learning framework is built to extract spatial structure feature. Finally, all the features are jointly learned and generate a structure-aware feature vector. We explore our method by using $\mathrm{C}++$, Matlab, TensorFlow platform, a serial of experimental analysis are carried out in the public 3D databases, they have shown that our deep features are stable for complex topological structure and large-scale geometric deformation. Compared with related methods, this method has higher classification accuracy in 3D shape classification.
\end{abstract}

Key words: shape classification; structure-aware; convolutional neural networks; deep learning

随着三维建模技术、成像技术的不断发展，互 联网中三维模型的数据量与复杂度与日俱增, 面
向海量、异构和复杂三维模型的智能形状分析方法 的研究, 已经成为计算机图形学和计算机视觉领

收稿日期: 2020-03-19; 修回日期: 2020-10-07. 基金项目: 国家自然科学基金(61702246); 大连市科技创新基金(2019J12GX038). 韩丽(1973-), 女, 博士, 教授, 硕士生导师, 主要研究方向为计算机图形学、计算机视觉; 朴京钰 (1996一), 女, 硕士研究生, 主要研 究方向为计算机图形学; 兰鹏燕(1996一), 女, 硕士研究生, 主要研究方向为计算机图形学; 王晓旻(1996一), 女, 硕士研究生, 主要 研究方向为计算机图形学; 于冰(1988一), 男, 硕士研究生, 主要研究方向为计算机图形学; 佟宇宁(1995一), 女, 硕士研究生, 主要 研究方向为计算机图形学; 徐圣斯(1992-), 男, 硕士研究生, 主要研究方向为计算机图形学. 
域的重要研究内容.

传统的三维形状分析方法基于人工提取的几 何特征构建形状描述符, 不仅过程繁复耗时, 且局 限于特定的模型与特定的应用, 很难推广到大规 模的三维数据分析中. 深度学习方法采用数据驱 动的方式自动学习三维模型的语义特征, 其在二 维图像、语音识别和文本处理等方面得到了长足的 发展. 面向三维模型的深度学习方法研究也吸引 了越来越多学者的关注, 并取得了显著的成果. 整 体来看, 基于深度学习的三维模型分类算法可以 分为基于低层次特征、基于原始数据以及基于视图 特征 3 大类. 由于深度学习在图像处理中的成功应 用, 基于视图的深度学习算法可以更好地利用图 像处理优秀的深度学习模型以及图像数据训练资 源, 获得了令人瞩目的识别与分类效果.

\section{1 相关工作}

深度学习理论的发展及其在二维图像领域的 成功应用, 为三维形状的分析与识别提供了新思 路. 近年来, 基于深度学习模型的三维形状分析方 法, 取得了显著的研究成果. 按照特征提取的方式 不同, 三维形状深度学习方法可分为：(1) 基于人 工特征的学习方法. 其手动提取三维模型的低层 特征, 并采用深度学习框架自动学习高层特征. 通 常采用的低层特征有几何矩 ${ }^{[1]}$ 、多尺度热核特征 ${ }^{[2]}$ 、 尺度不变热核特征 ${ }^{[3]}$ 和波核特征 ${ }^{[4]}$ 等; 进而，通过 构造特征词袋模型 ${ }^{[5-8]}$ 及深度神经网络模型 ${ }^{[9-12]}$, 实现高级特征的提取和模型的分类. 然而，这类方 法依赖于低层特征的手动选择，局限了学习过程 的高效性. (2) 基于原始数据的深度学习方法. 其 依据三维模型的原始表示来设计深度学习网络, 基于体素的深度学习方法将体素化表示的模型转 化为三维张量, 通过构建的神经网络框架自动提 取特征. 3D ShapeNets ${ }^{[13]}$ 直接从体素化数据中学习 特征分布, 实现了模型的识别与修复. $\mathrm{FPNN}^{[14]}$ 提 出了场探测滤波器的思想来获取鲁棒的全局特征 表示. OctNet ${ }^{[15-16]}$ 采用了八叉树结构实现了三维数 据的深度学习及稀疏表示学习, 提高了形状识别 的精度. 然而, 此类方法存在存储与计算量大的缺 陷，难以实现高效、精准的形状识别. Qi 等 ${ }^{[17]}$ 提出 直接在点云数据上进行深度学习的方法, 通过提 取点云的全局特征来实现三维形状分类与分割. PointNet $+{ }^{[18]}$ 进一步将点集划分为重叠的局部区 域, 引人 sampling 和 grouping 的迭代思想, 解决
了点云模型的局部特征学习问题. 由于图结构既 可以表示点云模型也可以表示网格模型, 因此, 基 于图卷积神经网络的深度学习方法受到了广大学 者的关注. 其根据 $k$ 近邻的方式建立图结构，典型 的方法有双图卷积网络(dual graph convolutional networks, DGCN) ${ }^{[19]}$ 、三维图卷积网络 (3D graph neural networks, $3 \mathrm{DGNN})^{[20]}$. 然而, 基于原始数据 的深度学习方法虽然能够有效地避免结构转化过 程中信息丢失的问题，但是，由于三维模型的表示 多样性与结构复杂性, 且缺乏充足的三维训练数 据资源, 往往导致学习模型存在不稳定性与通用 性较差的问题. (3) 基于视图的深度学习算法. 通 过投影的方式将三维模型转换为一系列二维视图, 再采取深度学习方法提取图像特征作为形状描述 符. DeepPano ${ }^{[21]}$ 将三维图形转换成全景图, 建立卷 积神经网络(convolutional neural networks, CNN)框 架, 提取紧凑的特征描述. Sinha 等 ${ }^{[22]}$ 将三维模型 参数化到球面上, 通过主曲率、热核特征的分布, 获得几何图像. $\mathrm{Su}$ 等 ${ }^{[23]}$ 的 $\mathrm{CNN}$ 结构将模型的多个 二维视图通过并行卷积框架与最大池化转换为一 个紧凑的形状表示, 有效地提高了三维形状识别 的效率与精度. Guo 等 ${ }^{[24]}$ 则提出一种基于几何和投 影图像的多模态学习体系结构. Leng 等 ${ }^{[25]}$ 进一步 引人了得分单元用于衡量多视图的贡献权重, 提 高了形状分类的性能. 基于视图的深度学习算法 有效地保留了三维形状的原始信息, 同时充分利 用了二维图像的海量数据资源及性能优越的深度 学习模型, 在三维识别和分类中取得了显著的成 果. 然而, 此类方法在变换过程中改变了三维形状 的局部和全局结构, 在一定程度上降低了特征鉴别 力. 提高基于视图的三维形状学习模型的稳定性与 高效性已经成为近年来广大学者关注的热点问题.

\section{2 本文方法概述}

为了提高基于视图深度学习的特征识别能力, 本文提出了结构感知的 CNN 联合学习框架, 不仅 学习模型的内蕴几何结构, 而且学习模型的空间 形状特征, 并进行有效的特征融合, 进而输人到 Softmax 分类器, 实现三维模型的结构识别与形状 分类.

\section{1 几何形状描述}

特征袋 (bag of feature, BoF) 模型通过聚类方法 将三维模型表示为无序的码本分布, 建立了介于 低级和高级语义特征之间的一种视觉特征表示. 
BoF 描述符集成了一系列几何特征并有效地揭示 了模型的内在结构性, 从而在非刚性三维形状分 类和检索中获得了显著的效果 ${ }^{[6-7]}$.

本文充分利用 $\mathrm{BoF}$ 模型的优势，采用优化的 多尺度热核特征(heat kernel signature, HKS)构造具 有区别性的几何形状描述符.

HKS 描述了给定流形上光滑函数的最优基, 具有多尺度特征 ${ }^{[26]}$, 能够揭示丰富的局部几何信 息. 其描述了表面上相对于时间的热传导, 热核公 式为

$$
K_{t}(x, x)=\sum_{i=0}^{m} \exp \left(-\lambda_{i} t\right) \boldsymbol{\phi}_{i}^{2}(x)
$$

其在离散空间通常由拉普拉斯算子(Laplace-Beltrami operator, LBO)的前 $m$ 个特征值 $\lambda_{i}$ 和特征向量 $\boldsymbol{\phi}_{i}$ 定 义. 可见, 三维模型的热核特征通过截取任意维度 的 LBO 特征向量来表示. 然而，不适当的特征向 量截取往往会降低特征的表示能力. 为了减少 HKS 特征提取过程的损失, 基于 Aflalo 等 ${ }^{[27]}$ 方法, 本文利用主成分分析(principal component analysis, PCA)与离散热核方程, 构建了特征嵌人空间与模 型空间的最优表示, 有效地提高了 LBO 嵌人空间 的表示能力 ${ }^{[28], \text { 如 }}$

$$
\left\{\begin{array}{l}
\boldsymbol{M}=(1-\mu) \boldsymbol{A} \boldsymbol{X} \boldsymbol{X}^{\mathrm{T}} \boldsymbol{A}=\mu \tilde{\boldsymbol{W}}_{h}^{-1} \\
\boldsymbol{W}_{h}=\frac{1}{4 \pi t^{2}} \mathrm{e}^{\frac{d\left(v_{i}, v_{j}\right)}{4 t}}
\end{array}\right.
$$

其中, 第 1 部分为 PCA 模型空间映射, $\boldsymbol{A}$ 是对角矩 阵, $A_{i i}$ 元素是共享顶点 $x_{i}$ 的所有三角形的面积之 和; $\boldsymbol{X}$ 是顶点的矩阵. 第 2 部分代表特征嵌人空 间, $\boldsymbol{W}_{h}$ 为相似度矩阵, $d\left(v_{i}, v_{j}\right)$ 表示任意一对网格 顶点之间的测地线距离; $0 \leqslant \mu \leqslant 1$ 是 PCA 映射与 特征映射的平衡因子。通过奇异值分解矩阵 $\boldsymbol{M}=\boldsymbol{Q} \boldsymbol{\Lambda} \boldsymbol{Q}^{\mathrm{T}}$ ，获得优化的特征值对角矩阵 $\boldsymbol{\Lambda}$ 与正 交的特征基矩阵 $\boldsymbol{Q}$.

为了评估优化特征空间的表示能力, 引人重 建误差

$$
E_{\mathrm{M}}=\underset{\hat{\boldsymbol{D}}}{\arg \min }\|\boldsymbol{X}-\hat{\boldsymbol{D}}\|_{\mathrm{F}}
$$

其中， $\hat{\boldsymbol{D}}=\boldsymbol{Q}_{m} \boldsymbol{Q}_{m}^{\mathrm{T}} \boldsymbol{X}$ 基于前一个特征正交基重构的 模型.

图 1 中分别对比了标准的 LBO 特征, Aflalo 等 ${ }^{[27]}$ 方法以及本文优化的 LBO 特征. 由特征可视化以 及基于前 $m=100$ 个特征向量所重构的模型结果 可见, 本文优化的特征向量具有较强的结构区分性, 有效地减少了特征损失, 提高了特征表示能力.

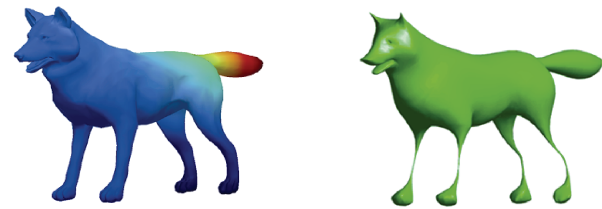

a. 基于标准 $\mathrm{LBO}$ 的特征分布及重建模型

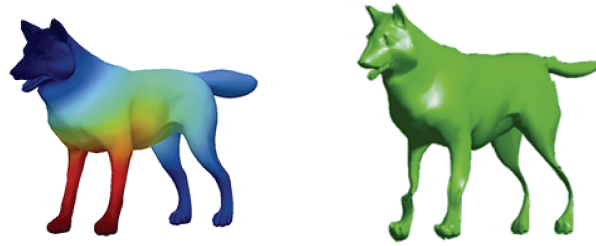

b. 基于Aflalo等 ${ }^{[27]}$ 方法的特征分布及重建模型
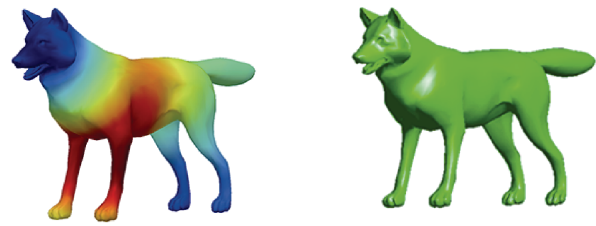

c. 优化的LBO $(\mu=0.5)$ 的特征分布及重建模型

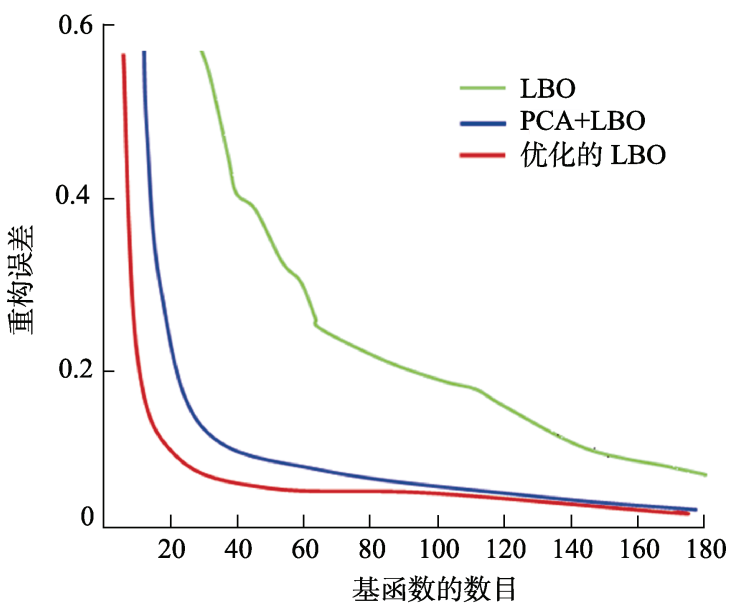

d. 不同嵌人方法的重构误差

图 1 基于不同 $\mathrm{LBO}$ 方法的特征分布和 重建模型及重构误差

进而, 基于优化的特征值与特征向量, 采用对 数区间 $\left[t_{\text {min }}=4 \ln 10 / \lambda_{300}, t_{\text {max }}=4 \ln 10 / \lambda_{2}\right]$ 设置时间 参数 $t$ 并划分 $p=100$ 个时间区，选择 $p$ 个特征值 和特征向量, 生成 $p$ 维的优化 HKS 特征表示.

因此，任意的三维模型 $M$ 可表示为几何特征 矩阵 $\boldsymbol{S}_{\mathrm{HKS}}=\left(s_{1}^{h}, s_{2}^{h}, \cdots, s_{n}^{h}\right), s_{i}^{h}$ 为任意顶点 $x_{i}$ 的多 尺度热核描述符, $n$ 为网格顶点的数量.

本文将提取的低层 HKS 几何特征嵌人词汇空 间中. 首先, 采用无监督 $k$-means 方法将几何特征 聚类生成 $k$ 个簇中心 (即码字), 构成码本; 其次, 利用软量化 (soft vector quantization, SVQ) ${ }^{[5]}$ 方法, 每个特征通过软分配矩阵映射到码本中进行特征 量化，其定义为 


$$
u_{i}=\frac{\exp \left(-\alpha\left\|s_{i}-v_{t}\right\|^{2}\right)}{\sum_{t=1}^{k} \exp \left(-\alpha\left\|s_{i}-v_{t}\right\|_{2}^{2}\right)}
$$

其中, $\|\cdot\|_{2}^{2}$ 表示 $L_{2}$ 范数; $\alpha$ 是控制软分配的平滑参 数, 取 $\alpha=1 /\left(8 \eta^{2}\right) ; \eta$ 表示聚类中心的平均值; $v_{t}$ 为 $k$ 个聚类中心; 每个局部描述符 $s_{i}$ 映射到码本进 行编码, 构造 $k \times n$ 维矩阵 $\boldsymbol{U}$. 最终, 将码本的分 布频率作为特征描述符, 即模型的 BoF 特征表示.

图 2 所示为以虎模型为例提取几何特征 HKS, 并通过 $k=20$ 均值聚类将其嵌人到词汇空间, 最 终获得码本的频率分布. 与标准的 HKS-BoF 结果 相比, 本文优化的 HKS-BoF 特征具有较好的区域
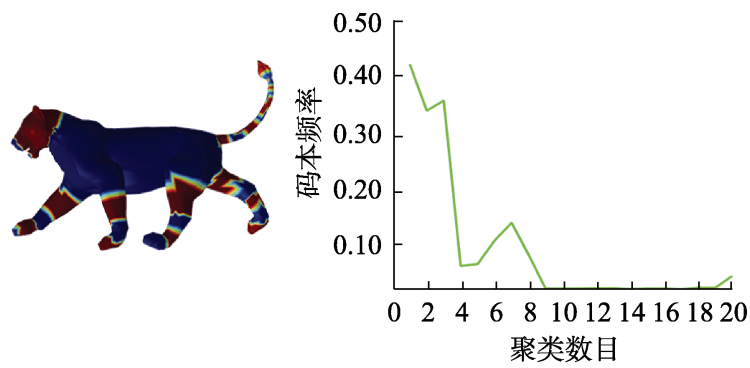

a. 基于标准 $\mathrm{HKS}$ 特征

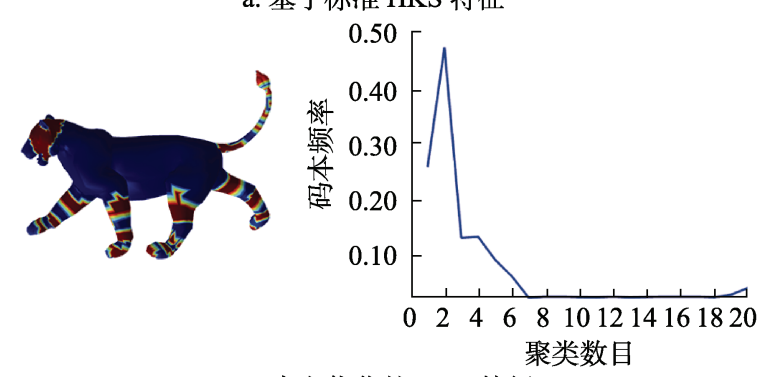

b. 本文优化的 HKS 特征
识别能力.

然而, BoF 局限于局部特征的统计, 忽略了特 征的空间关系. $\mathrm{SA}-\mathrm{BoF}^{[11]}$ 和 $\mathrm{GA}-\mathrm{BoF}^{[7]}$ 引入测地线 核函数距离以及双调和距离思想, 构建了形状上 下文信息, 生成具有区分性的全局 BoF 特征表示.

测地线核函数距离表示为

$$
\left\{\begin{array}{l}
g_{i j}=\sum_{x_{j} \in X} \sum_{x_{j} \in X} \phi_{l}(i) \phi_{l}(j) \exp \left(-k_{\mathrm{gd}} \frac{g\left(x_{i}, x_{j}\right)}{\sigma_{\mathrm{gd}}}\right) \\
\boldsymbol{F}=\boldsymbol{U} \boldsymbol{G} \boldsymbol{U}^{\mathrm{T}}
\end{array}\right.
$$

其中, 测地线核函数 $g_{i j}$ 是在一定距离范围内构建 的几何特征的分布信息; $\sigma_{\mathrm{gd}}$ 表示顶点间最大的测 地线距离; $k_{\mathrm{gd}}$ 为距离的衰减率.

尽管测地线距离具有等距不变性，在非刚性 三维形状匹配和检索中具有显著优势, 但是其往 往对拓扑噪声敏感.

双调和距离 ${ }^{[11]}$ 不仅对噪声和拓扑变化具有鲁 棒性，还具有全局形状感知和光滑性，

$$
\left\{\begin{array}{l}
k_{i j}=\sum_{l=1}^{m} \frac{1}{\lambda_{l}^{2}}\left(\varphi_{l}(i)-\varphi_{l}(j)\right)^{2} \\
\boldsymbol{F}=\boldsymbol{U} \boldsymbol{K} \boldsymbol{U}^{\mathrm{T}}
\end{array}\right.
$$

双调和距离 $k_{i j}$ 由任意一对网格顶点 $v_{i}$ 和 $v_{j}$ 之间的 LBO 的特征值和特征函数定义.

图 3 所示为以大卫人体模型为例，分别基于 $\mathrm{GA}-\mathrm{BoF}{ }^{[7]}$ 方法与 $\mathrm{SA}-\mathrm{BoF}^{[11]}$ 方法构造特征矩阵表 示, 从其重构的误差曲线可见 $\mathrm{SA}-\mathrm{BoF}^{[11]}$ 描述符表 现出更强的表示能力和更小的错误率.
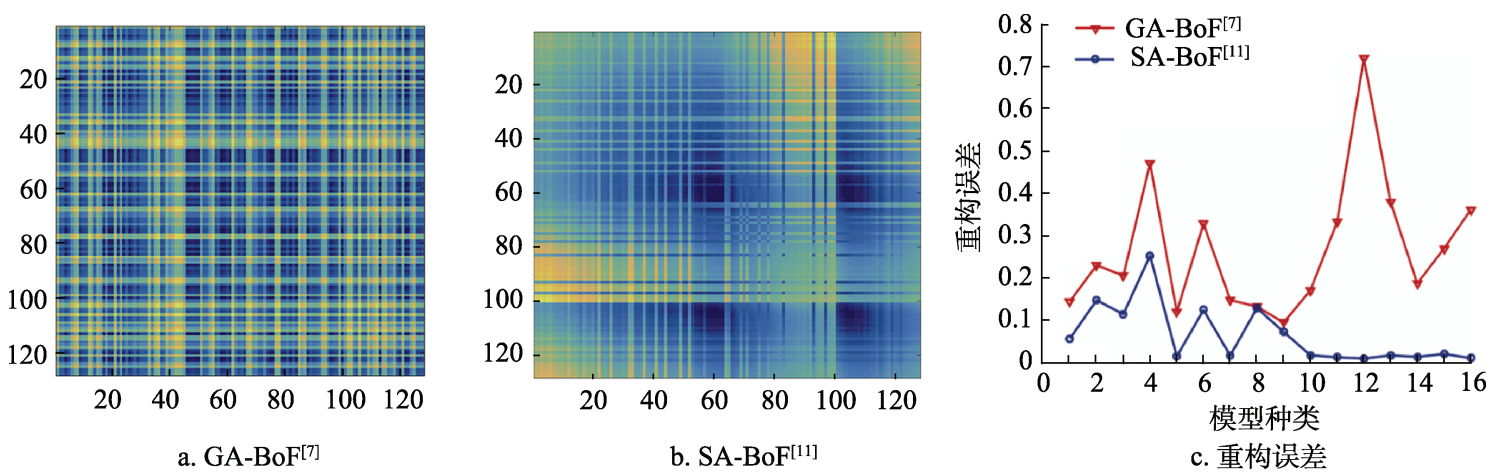

图 3 不同方法构造特征矩阵表示及重构误差

因此, 本文采用双调和距离, 基于优化的 HKS-BoF 特征, 有效地建立了优化的 BoF 特征表 示. 图 4 所示为针对 SHREC2016 库中人体模型所 构建的全局 $\mathrm{BoF}$ 特征矩阵, 可见, 本文 $\mathrm{BoF}$ 特征有 效地揭示了非刚性变换以及不完整模型中的内在
几何结构, 具有极强的稳定性.

\section{2 结构感知的联合学习模型}

$\mathrm{BoF}$ 图像虽然有效地揭示了模型的内蕴几何 特征, 但在一定程度上忽略了模型的空间结构信 息, 对于大尺度拓扑变换的模型缺乏鲁棒性. 为了 

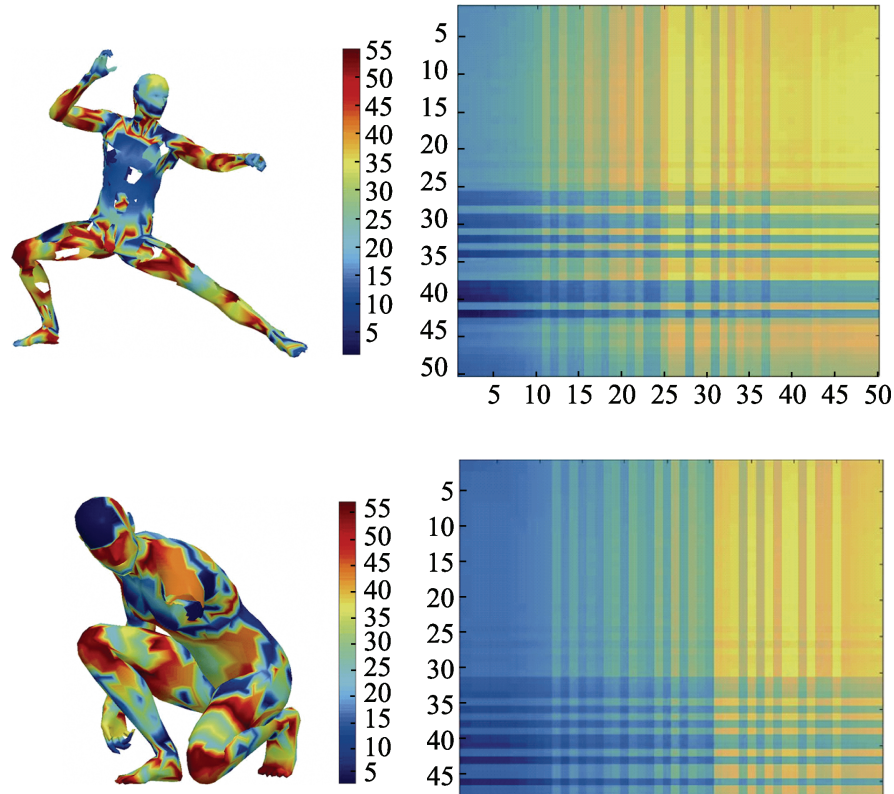

a. SHREC2016 库人体模型

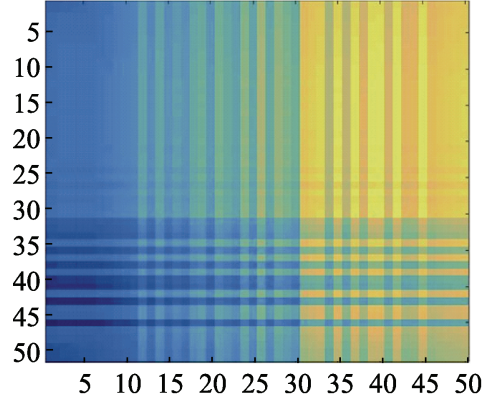

b. BoF 特征矩阵
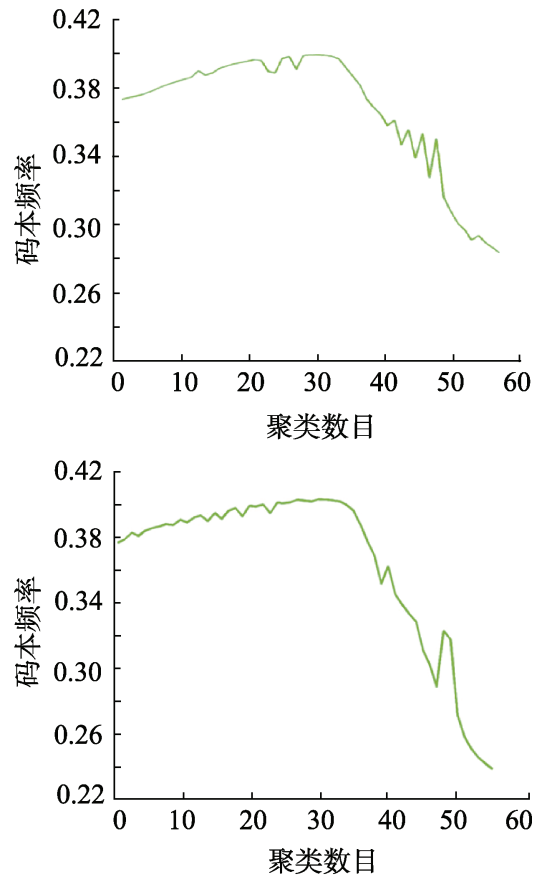

c. BoF 特征分布

图 4 基于全局优化的 $\mathrm{BoF}$ 特征及分布

有效提高深层特征的结构识别能力, 本文提出了 基于卷积神经网络的联合学习模型.

图 5 将生成的 $\mathrm{BoF}$ 特征输人到 $\mathrm{CNN}$ 框架中学 习模型的深度几何结构, 同时, 建立视图 CNN 框 架从多角度视图中学习模型的空间结构特征, 进 而, 联合优化学习生成结构感知的深度特征表示.

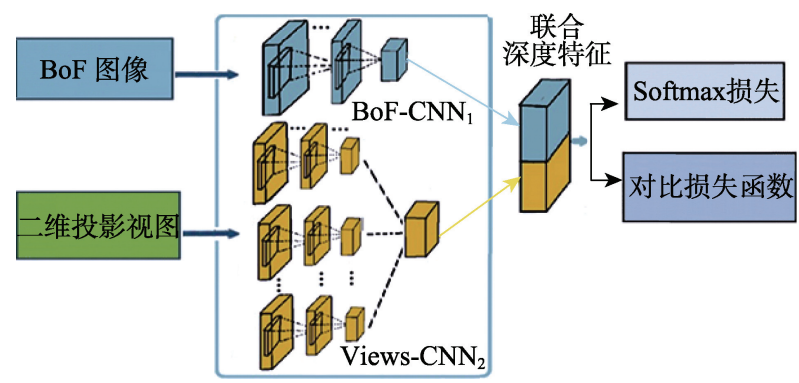

图 5 结构感知的 CNNs 联合学习框架

本文 BoF 卷积学习模型借鉴了 VGG-M 结构, 主要包括 5 个卷积层及 2 个全连接层. 每个卷积层 包含 ReLU 激活函数和 Max-Pooling 操作; 其中, 各 卷积层的卷积核依次设置为 $64,128,128,512,512$ 个, 所有卷积核大小均为 $3 \times 3$ 像素; 最后的全连接 层将产生 1024 维的特征向量.

多视图学习框架遵循 $\mathrm{MVCNN}^{[23]}$ 的成功思想, 首先将三维模型转换为多角度投影图像，并采用 并行的 CNN 框架, 分别从各个视图中提取特征, 最终, 通过 View-Pooling 操作融合为统一的深度 特征表示.
最终, 将 BoF-CNN 输出的内蕴结构特征与 Views-CNN 输出的空间结构特征进行有效连接, 通过损失函数优化学习生成信息丰富的结构特征, 并将其输人到 Softmax 分类器中进行形状识别与 分类.

为了提高 $\mathrm{CNN}$ 联合模型的学习性能, 实现类 间距离最大化和类内距离最小化的目标，本文采 用交叉熵与对比损失目标函数

$$
\left\{\begin{array}{l}
\min (L)=\min \left(L_{\mathrm{s}}+L_{\mathrm{c}}\right) \\
L_{\mathrm{c}}=\sum_{i=1}^{m / 2}\left(\alpha{D_{W}}^{2}+(1-\alpha) \max \left(T_{\mathrm{r}}-D_{W}, 0\right)^{2}\right)
\end{array}\right.
$$

以有效地提高学习模型的训练优化过程. 其中, $L_{\mathrm{s}}$ 为交叉熵损失函数; $L_{\mathrm{c}}$ 表示对比损失函数; $m$ 为数 据库中样本个数; $c$ 是样本中的类别数; $D_{W}$ 表示成 对的形状特征的 $L_{2}$ 范数; $\alpha$ 表示它们之间的相似 性，如果它们匹配，设置为 1 , 否则设置为 $0 ; T_{\mathrm{r}}$ 表 示不同类别的形状特征之间的距离. 对于不同的 特征，本文仅考虑 0 和 $T_{\mathrm{r}}$ 这 2 个欧几里得度量; 对 比损失函数 $L_{\mathrm{c}}$ 表示成对样本的匹配度, 它通过缩 小类内距离来提高特征的内聚性. 良好的损耗收 玫将有效地减少计算量, 有效地提高 CNN 框架的 学习效率.

表 1 所示为本文的结构感知 CNN 联合学习框 架的具体结构. 
表 1 不同网络结构的特征提取

\begin{tabular}{cl}
\hline 方法 & \multicolumn{1}{c}{ 特征提取 } \\
\hline \multirow{2}{*}{ BoF-CNN 1} & $\mathrm{C}(64) \mathrm{P}(2)-\mathrm{C}(128) \mathrm{P}(2)-\mathrm{C}(256) \mathrm{P}(2)-\mathrm{C}(512) \mathrm{P}(2)-$ \\
& $\mathrm{C}(512) \mathrm{P}(2)-\mathrm{Fc}(1024)-\mathrm{Fc}(1024)$ \\
Views-CNN & $\mathrm{C}(64) \mathrm{P}(3)-\mathrm{C}(128) \mathrm{P} 3-\mathrm{C}(256) \mathrm{P}(3)-\mathrm{C}(512) \mathrm{P} 3-$ \\
& $\mathrm{C}(512) \mathrm{P}(3)-\mathrm{Fc}(4096)-\mathrm{Fc}(4096)$ \\
\hline
\end{tabular}

本文具体算法步骤如下:

输人. 三维非刚性模型 $D=\left\{M_{1}, \cdots, M_{m}\right\}$, 初始 化参数与学习率 $\lambda_{t}$.

输出. 训练网络参数及分类结果.

Step1. 几何形状特征表示：

for $i=1: m$

计算模型 $M_{i}$ 的优化 HKS 特征矩阵 $s_{i}^{h}$,

基于 $k$-means 建立词典与特征矩阵 $\boldsymbol{U}_{i}$

计算双调和距离矩阵 $\boldsymbol{K}$, 生成 $\mathrm{BoF}$ 矩阵 $\boldsymbol{F}_{i}$ end for

计算 $\mathrm{BoF}$ 矩阵转换为 $\mathrm{BoF}$ 图像 $B_{i}(i=1,2, \cdots, m)$.

Step2. 多视图生成:

for $i=1: m$

生成模型 $M_{i}$ 的视图 $V_{i}^{t}(t=1,2, \cdots, 12)$

end for

Step3. 结构感知联合学习模型的优化训练:

while $(j \leqslant T)$ do

Step3.1. 输人 $\mathrm{BoF}$ 特征图像 $B_{i}$ 到 $\mathrm{CNN}_{1}$, 学习 并提取 1024 维特征 $f\left(B_{i}\right)$;

Step3.2. 输人视图 $V_{i}^{t}$ 到 $\mathrm{CNN}_{2}$, 并提取 4096 维特征 $f\left(V_{i}\right)$;

Step3.3. 建立联合深度特征 $f(V B)_{i}$, Softmax 层进行分类预测 $\hat{p}_{i}$ ，统计分类精度 $\operatorname{Acc}(i)$ ；

Step3. 4. 通过前向传播计算交叉嫡与对比损耗;

Step3.5. 通过反向传播计算 $\partial L_{W} / \partial D_{W}$, 更新

$\mathrm{CNN}_{1}$ 和 $\mathrm{CNN}_{2}$ 参数, $j++$;

end while

Step4. 输出最终训练分类精度 $\operatorname{Acc}(i)$.

\section{3 实 验}

采用经典的非刚性三维数据库 SHREC2010, SHREC2011 和 SHREC2015 对本文方法进行实验 分析与论证. 其中, SHREC2010 包含来自 10 个类 别的 200 个不同姿势的非刚性模型; SHREC2011 包 含 30 个不同种类的 600 个网格模型; SHREC2015 拥有 50 个类别的 1200 个网格模型. 在实验中，通 过随机选择 $80 \%$ 的模型作为训练集, $20 \%$ 的模型作 为测试集.

由于本文使用的学习模型采用对比损失与交 叉熵损失联合目标函数，因此，在训练阶段，将训
练数据成对组织输人, 每个数据生成 12 个投影视 图和 1 个 $\mathrm{BoF}$ 特征图像。

每对数据的投影视图被输人到并行的 Views$\mathrm{CNN}_{2}$ 框架, 通过最大池化学习深层的视图特征. 同时, 将每对 BoF 图像发送到 BoF-CNN $\mathrm{CN}_{1}$, 提取深 层的结构特征. 进而, 将所有深层特征进行连接, 最终生成结构特征表示, 通过 Softmax 分类器实现 三维形状的识别与分类.

本文的网络参数使用联合目标函数和随机梯 度下降进行了优化, 其中, 学习率设为 0.03 , 学习 率衰减设为 0.95 , dropout 为 0.5 , 正则化权重为 $5 \times 10^{-4}$, 所有模型都采用 1000 次迭代的训练.

\section{1 优化的几何结构特征}

首先，实验论证了 HKS-BoF 特征的稳定性与 识别性. 本文采用统一的 CNN 框架(VGG-M)在非 刚性模型 SHREC 数据集上分别对比了 AGD-BoF, HKS-BoF 以及优化 HKS-BoF 的识别精度, 如表 2 所示.

以 SHREC2010 库为例, 各个类在不同 BoF 特 征上的识别精度曲线如图 6a 所示. 其中, AGD$\mathrm{BoF}$ 的区分性最弱, 本文的 BoF 特征在平均识别精 度上分别高于 AGD-BoF 以及标准的 HKS-BoF $10.00 \%$ 和 $6.00 \%$.

\begin{tabular}{|c|c|c|c|c|}
\hline \multirow{2}{*}{$\mathrm{BoF}$ 特征 } & \multicolumn{3}{|c|}{ SHREC } & \multirow{2}{*}{ mAP } \\
\hline & 2010 & 2011 & 2015 & \\
\hline AGD-BoF ${ }^{[7]}$ & 83.26 & 84.53 & 85.78 & 84.52 \\
\hline $\mathrm{HKS}-\mathrm{BoF}^{[5]}$ & 87.65 & 88.01 & 90.36 & 88.67 \\
\hline 本文 BoF & 93.78 & 95.52 & 95.41 & 94.90 \\
\hline
\end{tabular}

图 6b 所示为基于本文优化的 HKS-BoF 特征的 交叉混淆矩阵实验结果. 随机在每个类中选取 20 个样本进行分类测试统计, 其中绝大多数的模型 均能获得正确的识别结果, 仅仅在结构极为相似 的 crab, spider 和 plier 中出现了个别的错误.

\section{2 结构感知联合学习模型的形状分类性能}

本文提出了结构感知 CNN 联合学习框架, 不 仅提取模型的几何结构特征 (optimal HKS-BoF), 而且综合分析模型的空间结构特征(多视图投影), 建立有效的联合学习训练机制, 生成具有较强识 别力的形状表示, 有效地提高了三维非刚性模型 的结构识别与分类精度.

本文以典型的非刚性模型数据库 SHREC2010, SHREC2011 和 SHREC2015 中的模型为例, 进行了 学习框架的测试. 首先分别使用 BoF-CNN, Views- 


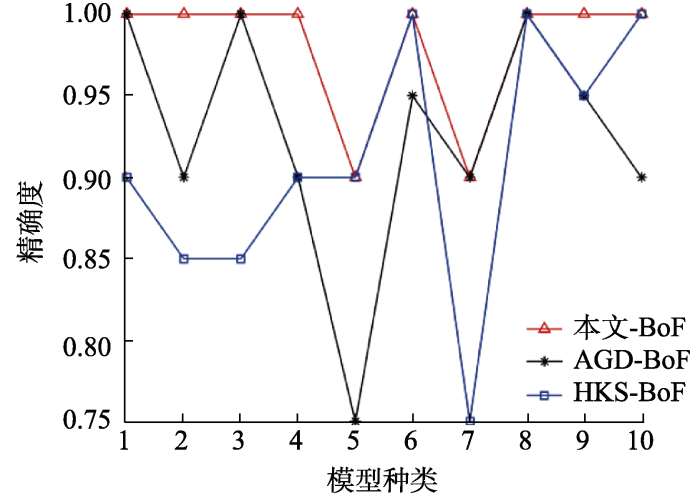

a. 识别精度

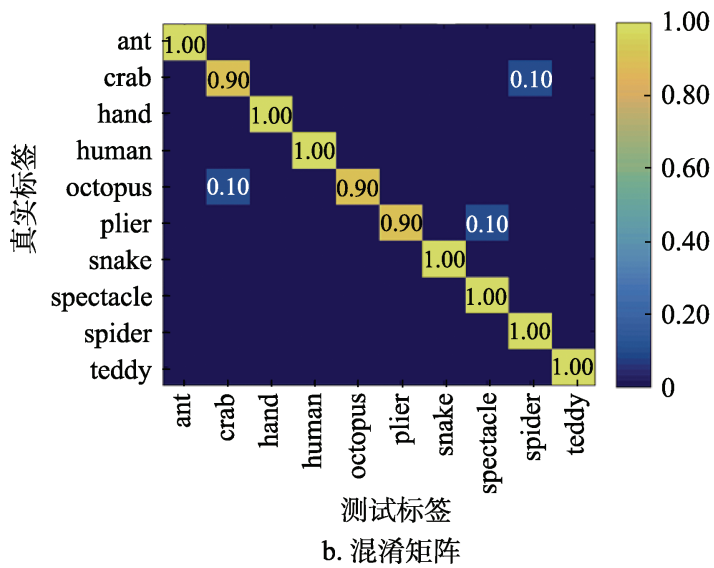

图 6 SHREC2010 数据库中各类识别精度与混淆矩阵

CNN 以及联合学习模型, 对各个类的模型进行了 测试, 对比分析如表 3 所示. 其中, 基于 BoF 几何 结构学习的识别效果较低, 而基于多视图学习的 结构识别效果平均高于 $\mathrm{BoF}$ 特征学习 $2.00 \%$, 与之 相比, 本文结构感知联合学习框架的精度分别高 于它们 $0.70 \%$ 与 $3.80 \%$, 有效地提高了非刚性三维 形状分类的性能.

表 3 基于不同 $\mathrm{CNN}$ 框架的分类精确度比较 \%

\begin{tabular}{lccc}
\hline \multirow{2}{*}{\multicolumn{1}{c}{ 方法 }} & \multicolumn{3}{c}{ SHREC } \\
\cline { 2 - 4 } & 2010 & 2011 & 2015 \\
\hline BoF-CNN & 93.78 & 95.52 & 95.41 \\
Views-CNN & 97.61 & 98.04 & 96.49 \\
结构感知 CNNs & 98.02 & 98.33 & 97.12 \\
联合学习模型 & & & \\
\hline
\end{tabular}

如图 7 所示, 基于不同的学习框架对 SHREC2011 库中各个类的分类性能进行了交叉验 证，图 7a 所示为基于 BoF, Views 以及基于联合 $\mathrm{CNN}$ 学习框架中各个类的识别精度曲线. 可见, 基于 BoF 的 $\mathrm{CNN}$ 学习框架在识别结构单一的模型 具有较差的识别精度.

图 7b 图 7d 为基于不同学习框架的混淆矩阵 测试结果. 随机在每个类别中选取 20 个样本进行
测试, 对角线代表识别正确的样本数目, 非对角线 上的数字代表错误分类的数目. 如图 7c 所示为基 于 $\mathrm{BoF}$ 学习框架的结果, 可见, 其对于几何结构不 明显的类别 two-balls, lamp, snake 等识别率较低; 而图 7d 为基于视图的识别, 其容易混淆外部形状 相似的模型, 如 man 和 gorilla, scissor 和 pliers. 而 本文的结构感知联合学习框架融合了外蕴与内蕴 的结构深度特征, 大大改善了在 birds, dogs, two-balls 等类别的识别能力(图 7b), 同时在相似结 构的 octopus, man, pilers 等分类中, 也显著提高了 性能.

\section{3 与相关工作的对比分析}

为了进一步验证本文结构感知联合学习模型 的高效性, 与先进的研究成果进行了性能比较. 如 表 4 所示, 首先与具有代表性的 BoF 方法进行了对 比分析. 其中, $\mathrm{GA}-\mathrm{BoF}^{[7]}$ 方法采用热核和 $\mathrm{AGD}$ 作 为低层描述符，利用测地线核函数构造全局 $\mathrm{BoF}$, 避免了时间和形状尺寸的影响, 并通过双层深度 信念网络(deep belief nets, DBN)学习深层特征; $\mathrm{SA}-\mathrm{BoF}^{[11]}$ 采用谱图小波作为几何特征, 再利用自 动编码器学习高级特征; $\mathrm{SGWC}-\mathrm{BoF}^{[9]}$ 利用谱图小 波特征构造中层特征，再利用多类支持向量机实 现分类任务.

\begin{tabular}{|c|c|c|c|c|}
\hline \multirow{2}{*}{ 方法 } & \multicolumn{3}{|c|}{ SHREC } & \multirow{2}{*}{$\mathrm{mAP}$} \\
\hline & 2010 & 2011 & 2015 & \\
\hline GA-BoF ${ }^{[7]}$ & 86.02 & 93.20 & 72.93 & 84.11 \\
\hline $\mathrm{SA}-\mathrm{BoF}^{[11]}$ & 91.83 & 98.00 & 84.27 & 91.37 \\
\hline SGWC-BoF ${ }^{[9]}$ & 95.66 & 97.66 & 92.54 & 95.28 \\
\hline $\mathrm{BoF}-\mathrm{CNN}$ & 93.75 & 95.50 & 95.47 & 94.91 \\
\hline DeepShape $^{[29]}$ & 95.50 & 96.53 & 92.87 & 94.96 \\
\hline DeepGM $^{[12]}$ & 96.33 & 97.89 & 93.03 & 95.75 \\
\hline FeaStNet $^{[30]}$ & 97.84 & 98.12 & 96.35 & 97.43 \\
\hline $\mathrm{MVCNN}^{[23]}$ & 97.42 & 97.86 & 96.17 & 97.15 \\
\hline 本文 CNN & 98.02 & 98.33 & 97.12 & 97.82 \\
\hline
\end{tabular}

本文 BoF-CNN 学习框架使用优化的多尺度 HKS 构造了具有较强辨别能力的 BoF 图像, 并联 合目标函数优化训练, 通过 CNN 提取内蕴的几何 结构特征; 其与 $\mathrm{GA}-\mathrm{BoF}^{[7]}$ 和 $\mathrm{SA}-\mathrm{BoF}^{[11]}$ 相比, 性能 平均分别提高了 $3.00 \%$ 到 $10.00 \%$, 略低于 SGWC$\mathrm{BoF}^{[9]} 0.30 \%$. 然而, 通过与视图特征的有效融合, 可以看到本文模型性能比 BoF-CNN 学习总体提高 了近 $4.00 \%$ 的识别精度, 而且比 SGWC-BoF ${ }^{[9]}$ 提高 了近 $3.00 \%$. 


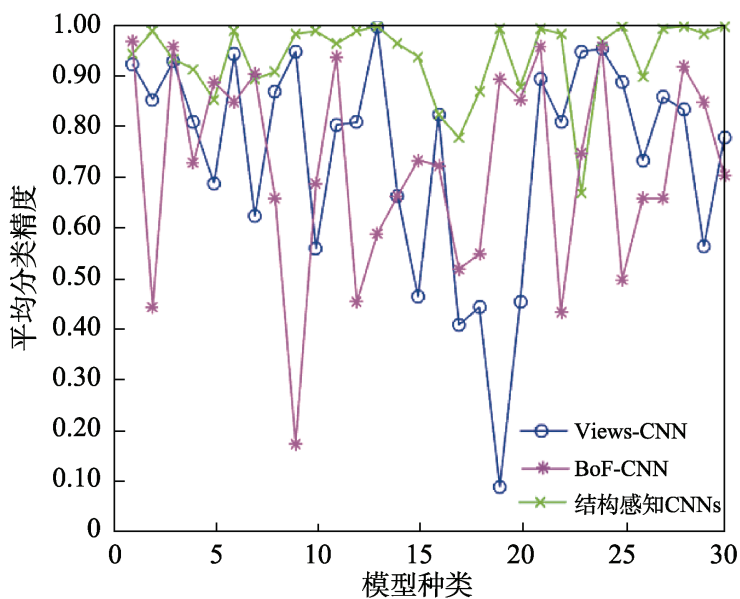

a. 基于2011库的3种CNN结构识别精度

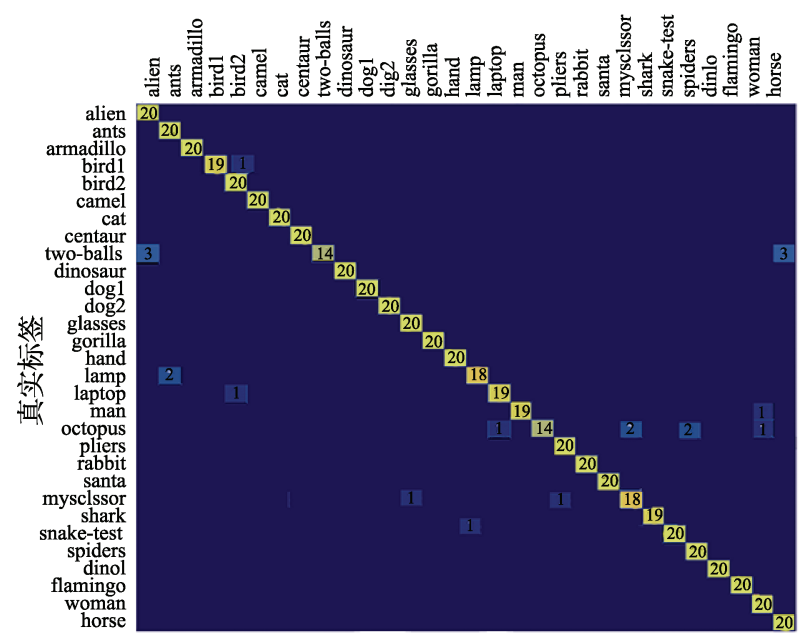

预测标签

c. 基于 $\mathrm{BoF}$ 的 $\mathrm{CNN}_{1}$

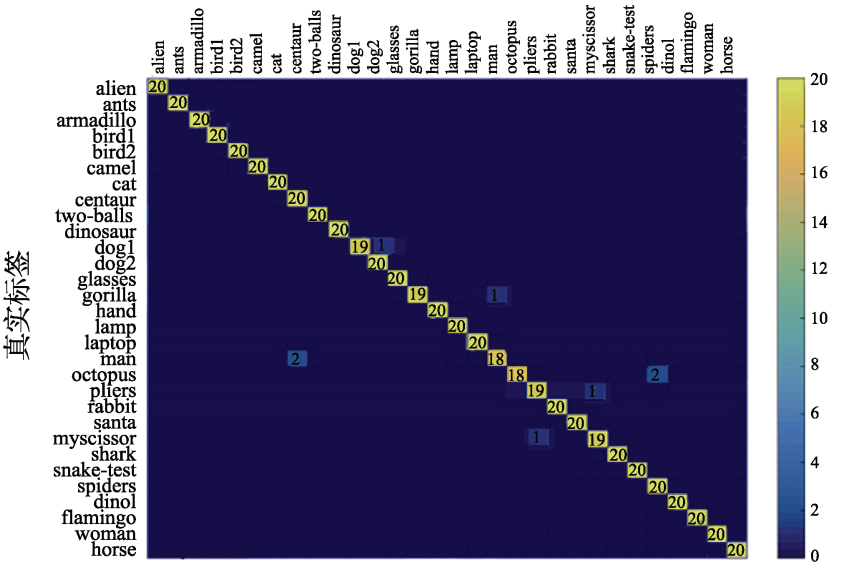

预测标签

b. 结构感知的 CNNs

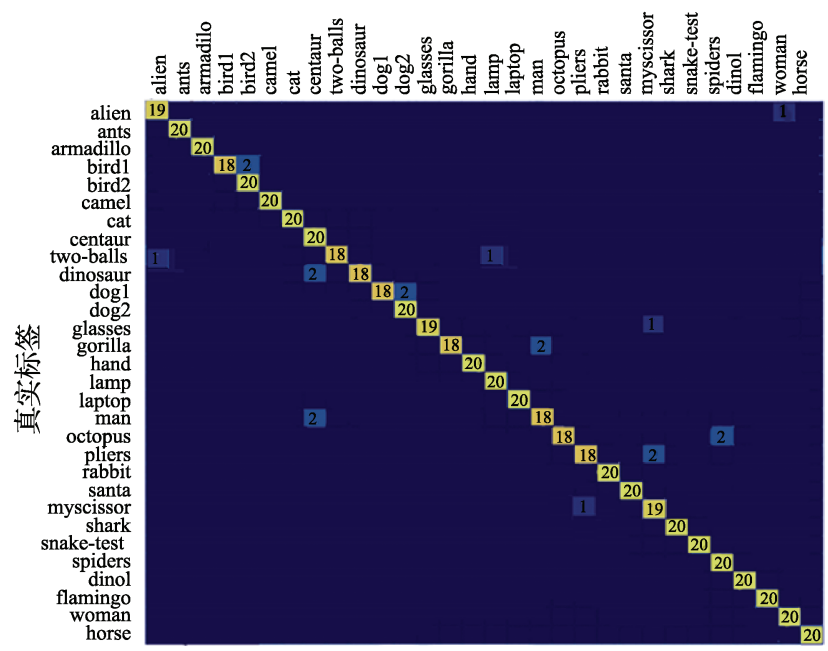

预测标签

d. 基于视图的 $\mathrm{CNN}_{2}$

图 7 数据库 SHREC2011 在不同学习框架的识别精度与混淆矩阵

图 8a 所示为本文方法与现有的 $\mathrm{BoF}$ 方法的精 确率-召回率(precision-recall，PR)曲线对比，本文 结构感知方法的平均检索精度比 SGWC-BoF $\mathrm{F}^{[9]}$ 略高 $0.80 \%$, 比 $\mathrm{GA}-\mathrm{BoF}^{[7]}$ 提高了 $6.20 \%$.

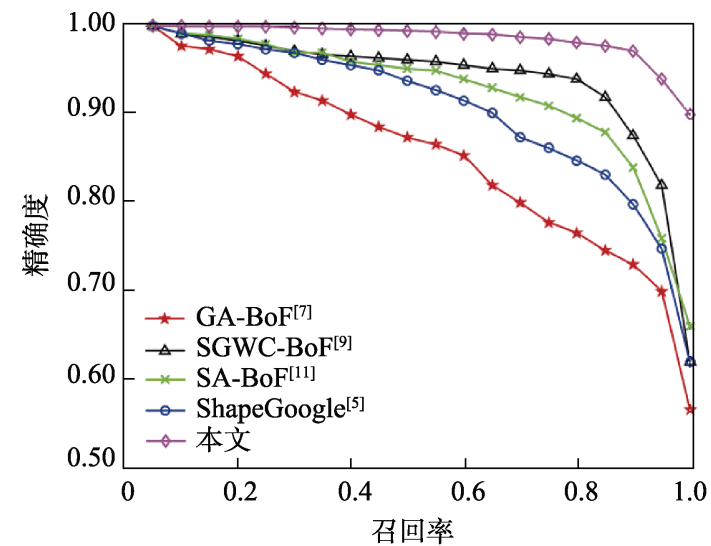

a. 不同的BoF方法
此外, 进一步与基于深度学习的三维形状分 析方法 MVCNN ${ }^{[23]}$, DeepGM ${ }^{[12]}$, DeepShape ${ }^{[29]}$ 以及 FeaStNet ${ }^{[30]}$ 进行了对比分析.

DeepShape ${ }^{[29]}$ 是以 HKS 形状描述符为输人,

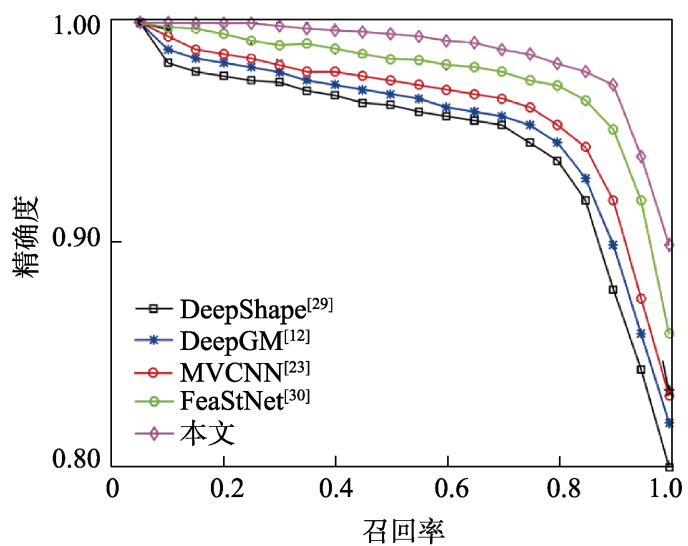

b. 不同的深度学习方法

图 8 不同方法 PR 曲线对比 
通过多对一编码的神经网络学习深度特征. $\mathrm{DeepGM}^{[12]}$ 以测地线距离为输人, 在自动编码器 学习框架中学习深层特征. FeaStNet ${ }^{[30]}$ 在局部邻域 中使用动态图卷积算子, 以原始三维形状坐标作 为输人学习局部形状属性. $\mathrm{MVCNN}^{[23]}$ 将三维形状 转化为多角度投影视图, 并采用并行 $\mathrm{CNN}$ 框架进 行特征聚合及细化.

由表 4 可见, 结合视图空间结构特征与 BoF 几 何结构特征，可大大提高形状分类的性能; 分别比 DeepGM $^{[12]}$ 和 DeepShape ${ }^{[29]}$ 平均高 $1.44 \%$ 和 $0.39 \%$, 比 $\mathrm{MVCNN}^{[23]}$ 高 $1.09 \%$. 由图 $8 \mathrm{~b}$ 所示对比 PR 曲线可 见，本文结构特征具有显著的区分性，平均检索精 度高于 $\mathrm{MVCNN}^{[23]} 2.60 \%$ ，高于 $\mathrm{FeaStNet}^{[30]} 1.20 \%$; 而对比 DeepGM ${ }^{[12]}$ 和 DeepShape ${ }^{[29]}$, 则提升了近 $3.40 \%$ 的平均检索精度.

\section{4 结 语}

随着互联网中三维模型的数据量与复杂度迅 猛增长, 面向海量、复杂三维模型的智能形状分析 方法需求日益紧迫. 本文充分分析了基于视图的 CNN 学习模型在三维形状识别中的优势与局限性, 提出了结构感知联合学习模型的三维形状识别方 法, 旨在提高视图特征学习的稳定性与高效性. 由 于多角度投影视图忽略了模型的内在几何结构信 息，因此从一定程度上降低了深度特征在形状识 别中的精确性.

本文提出了优化的 BoF 图像表示, 有效地揭 示了不同拓扑变化的非刚性模型的内在结构一致 性. 进而提出了基于几何结构学习与空间结构学 习的联合卷积网络框架，通过交叉熵与对比损失 函数联合学习机制，生成具有丰富信息的形状表 示，有效地提高了特征的区分性与鲁棒性，并通过 实验论证了其在非刚性三维形状分类中的优秀性能.

在未来的工作中，将深人探讨不同模态深度 特征的加权融合机制, 进一步研究大规模复杂点 云模型的形状分析与识别方法.

\section{参考文献(References):}

[1] Saupe D, Vranić D V. 3D model retrieval with spherical harmonics and moments[C] //Proceedings of the 23rd DAGM Symposium on Pattern Recognition. Heidelberg: Springer, 2001: 392-397

[2] Ovsjanikov M, Bronstein A M, Bronstein M M, et al. Shape Google: a computer vision approach to isometry invariant shape retrieval[C] //Proceedings of the IEEE Conference on Computer Vision and Pattern Recognition. Los Alamitos: IEEE Computer Society Press, 2009: 320-327

[3] Bronstein M M, Kokkinos I. Scale-invariant heat kernel signatures for non-rigid shape recognition[C] //Proceedings of the IEEE Conference on Computer Vision and Pattern Recognition. Los Alamitos: IEEE Computer Society Press, 2020: 1704-1711

[4] Aubry M, Schlickewei U, Cremers D. The wave kernel signature: a quantum mechanical approach to shape analysis[C] // Proceedings of the IEEE Conference on Computer Vision and Pattern Recognition. Los Alamitos: IEEE Computer Society Press, 2011: 1626-1633

[5] Bronstein A M, Bronstein M M, Guibas L J, et al. Shape Google: geometric words and expressions for invariant shape retrieval[J]. ACM Transactions on Graphics, 2011, 30(1): 1-20

[6] Litman R, Bronstein A, Bronstein M, et al. Supervised learning of bag-of-features shape descriptors using sparse coding[J]. Computer Graphics Forum, 2014, 33(5): 127-136

[7] Bu S H, Liu Z B, Han J W, et al. Learning high-level feature by deep belief networks for 3-D model retrieval and recognition[J]. IEEE Transactions on Multimedia, 2014, 16(8): 2154-2167

[8] Han Z Z, Liu Z B, Vong C M, et al. BoSCC: bag of spatial context correlations for spatially enhanced $3 \mathrm{D}$ shape representation[J]. IEEE Transactions on Image Processing, 2017, 26(8): $3707-3720$

[9] Masoumi M, Li C Y, Ben Hamza A. A spectral graph wavelet approach for nonrigid 3D shape retrieval[J]. Pattern Recognition Letters, 2016, 83(1): 339-348

[10] Ghodrati H, Ben Hamza A. Nonrigid 3D shape retrieval using deep auto-encoders[J]. Applied Intelligence, 2017, 47(1): 44-61

[11] Ghodrati H, Ben Hamza A. Deep shape-aware descriptor for non-rigid 3D object retrieval[J]. International Journal of Multimedia Information Retrieval, 2016, 5(3): 151-164

[12] Luciano L, Ben Hamza A. Deep learning with geodesic moments for 3D shape classification[J]. Pattern Recognition Letters, 2018, 105(1): 182-190

[13] Wu Z R, Song S R, Khosla A, et al. 3D ShapeNets: a deep representation for volumetric shapes[C] //Proceedings of the IEEE Conference on Computer Vision and Pattern Recognition. Los Alamitos: IEEE Computer Society Press, 2015: 1912-1920

[14] Li Y Y, Pirk S. FPNN: field probing neural networks for 3D data[C] //Proceedings of the Advances in Neural Information Processing Systems. New York: ACM Press, 2016: 307-315

[15] Riegler G, Ulusoy A O, Geiger A. OctNet: learning deep 3D representations at high resolutions[C]//Proceedings of the IEEE Conference on Computer Vision and Pattern Recognition. Los Alamitos: IEEE Computer Society Press, 2017: 21-26

[16] Wang P S, Sun C Y, Liu Y, et al. Adaptive O-CNN: a patch-based deep representation of 3D shapes[J]. ACM Transactions on Graphics, 2018, 37(6): 1-11

[17] Qi C R, Su H, Mo K C, et al. PointNet: deep learning on point sets for 3D classification and segmentation[C] //Proceedings of the IEEE Conference on Computer Vision and Pattern Recognition. Los Alamitos: IEEE Computer Society Press, 2017: $77-85$ 
[18] Qi C R, Yi L, Su H, et al. PointNet++: deep hierarchical feature learning on point sets in a metric space[OL]. [2020-03-19]. https://arxiv.org/abs/1706.02413

[19] Zhuang C Y, Ma Q. Dual graph convolutional networks for graph-based semi-supervised classification[C] //Proceedings of the World Wide Web Conference. New York: ACM Press, 2018: 499-508

[20] Qi X J, Liao R J, Jia J Y, et al. 3D graph neural networks for RGBD semantic segmentation[C] //Proceedings of the IEEE International Conference on Computer Vision. Los Alamitos: IEEE Computer Society Press, 2017: 5209-5218

[21] Shi B G., Bai S, Zhou Z C, et al. DeepPano: deep panoramic representation for $3 \mathrm{D}$ shape recognition[J]. IEEE Signal Processing Letters, 2015, 22(12): 2339-2343

[22] Sinha A, Bai J, Ramani K. Deep learning 3D shape surfaces using geometry images[C] //Proceedings of the European Conference on Computer Vision. Heidelberg: Springer, 2016: 223-240

[23] Su H, Maji S, Kalogerakis E, et al. Multi-view convolutional neural networks for $3 \mathrm{~d}$ shape recognition[C] //Proceedings of the IEEE International Conference on Computer Vision. Los Alamitos: IEEE Computer Society Press, 2015: 7-13

[24] Guo H Y, Wang J Q, Gao Y, et al. Multi-view 3D object re- trieval with deep embedding network[J]. IEEE Transactions on Image Processing, 2016, 25(12): 5526-5537

[25] Leng B, Zhang C, Zhou X C. Learning discriminative 3D shape representations by view discerning networks[J]. IEEE Transactions on Visualization and Computer Graphics, 2019, 25(10): 2896-2909

[26] Sun J, Ovsjanikov M, Guibas L J. A concise and provably informative multi-scale signature based on heat diffusion[J]. Computer Graphics Forum, 2009, 28(5): 1383-1392

[27] Aflalo Y, Brezis H, Kimmel R. On the optimality of shape and data representation in the spectral domain[J]. SIAM Journal on Imaging Sciences, 2015, 8(2): 1141-1160

[28] Han L, Yu B, Piao J Y, et al. Multi-channel joint sparse learning model for non-rigid three-dimensional object classification[J]. Journal of Imaging Science and Technology, 2020, 63(3): 1-11

[29] Xie J, Fang Y, Zhu F. DeepShape: deep learned shape descriptor for 3D shape matching and retrieval[C] //Proceedings of the IEEE Conference on Computer Vision and Pattern Recognition. Los Alamitos: IEEE Computer Society Press, 2015: 7-12

[30] Verma N, Boyer E, Verbeek J. FeaStNet: feature-steered graph convolutions for $3 \mathrm{D}$ shape analysis[C] //Proceedings of the IEEE Conference on Computer Vision and Pattern Recognition. Los Alamitos: IEEE Computer Society Press, 2018: 18-23 